\title{
Glycerol catabolism in Aspergillus nidulans
}

\author{
Dirk H. A. Hondmann, Ronald Busink, Cor F. B. Witteveen and JaAP Visser* \\ Department of Genetics, Section Molecular Genetics, Agricultural University, Dreyenlaan 2, 6703 HA Wageningen, \\ The Netherlands
}

(Received 31 August 1990; revised 26 November 1990; accepted 11 December 1990)

\begin{abstract}
Glycerol is catabolized in Aspergillus nidulans by glycerol kinase and a mitochondrial FAD-dependent $s n$-glycerol3-phosphate dehydrogenase. The levels of both enzymes are controlled by carbon catabolite repression and by specific induction. Biochemical and genetical analyses show that dihydroxyacetone and D-glyceraldehyde are converted into glycerol and then catabolized by the same pathway. D-Glyceraldehyde can be reduced by NADP+dependent glycerol dehydrogenase or by alcohol dehydrogenase I, while dihydroxyacetone is only reduced by the first enzyme. Three new glycerol non-utilizing mutants have been found. These three mutations define three hitherto unknown loci, $g l c E, g l c F$ and $g l c G$. The mutation in $g l c G$ leads to a greatly decreased sn-glycerol-3phosphate dehydrogenase activity.
\end{abstract}

\section{Introduction}

Glycerol is one of the polyols that is formed rapidly by Aspergillus nidulans when this fungus is grown on glycolytic carbon sources, especially under strong oxygenation (Dijkema et al., 1985). Upon starvation, glycerol is the first polyol to disappear. Glycerol plays an important role in several physiological processes. Beever \& Laracy (1986) showed that in $A$. nidulans glycerol and erythritol are the major osmoregulatory solutes. On hyperosmotic growth media both polyols accumulate, whereas they decrease rapidly in amount after transfer to a hypoosmotic medium. Glycerol formation is also one of the early events during germination of fungal spores (Van Laere \& Hulsmans, 1987). In the stage just before citric acid production by Aspergillus niger glycerol has been reported to accumulate (Legisa and Mattey, 1986). Glycerol is catabolized again when citric acid production starts. This led Legisa \& Mattey (1986) to suggest that glycerol accumulation may even initiate citric acid production in $A$. niger. The catabolism of dihydroxyacetone (DHA) (Schuurink et al., 1990) and in part that of D-galacturonate (Uitzetter et al., 1986) are also likely to proceed through glycerol in $\boldsymbol{A}$. nidulans.

Glycerol catabolism has been studied in many organisms, as reviewed by $\operatorname{Lin}(1976,1977)$. In most

Abbreviations: ADH, alcohol dehydrogenase; DHA, dihydroxyacetone; DHAP, dihydroxyacetone phosphate; D-GAD, D-glyceraldehyde; G3P, glycerol 3-phosphate; MTT, 3-(4,5-dimethylthiazolyl2)2,5-diphenyltetrazolium bromide. organisms, both prokaryotes and eukaryotes, glycerol utilization is initiated by phosphorylation followed by oxidation of glycerol 3-phosphate (G3P) to dihydroxyacetone phosphate (DHAP) by G3P dehydrogenase. This enzyme is membrane-associated in prokaryotes and located on the inner membrane of mitochondria in eukaryotes. Three alternative pathways have been found. One pathway involves an $\mathrm{NAD}^{+}$-dependent glycerol dehydrogenase that converts glycerol into DHA, which is then phosphorylated to DHAP by DHA kinase (May \& Sloan, 1981). This pathway can be used by several bacteria and by some yeasts. In the second alternative pathway glycerol is converted to glyceraldehyde by an $\mathrm{NADP}^{+}$-dependent glycerol dehydrogenase (Viswanath-Reddy et al., 1978). The third alternative, found amongst others in Aspergillus japonicus, involves glycerol oxidase (Uwajima et al., 1980). This enzyme oxidizes glycerol, using molecular oxygen, to D-glyceraldehyde (D-GAD) and hydrogen peroxide without mediation of NAD(P)+ (Uwajima et al., 1990).

Four glycerol non-utilizing mutant classes have been reported thus far for $\boldsymbol{A}$. nidulans. glc $\boldsymbol{A}$ mutations were proposed to lead to glycerol kinase deficiency, $g l c B$ and glcD strains are deficient for the mitochondrial glycerol3-phosphate dehydrogenase (Payton, 1978; Uitzetter, 1982; Arst et al., 1990) and glcC strains are defective in glycerol uptake (Visser et al., 1988) A. nidulans is therefore likely to use the phosphorylating pathway.

We present here a more detailed study of glycerol catabolism in $A$. nidulans and describe three new 
independent glycerol non-utilizing mutants. The effect of various carbon sources on glycerol catabolism in wildtype and glc mutants has also been investigated to initiate studies on regulation.

\section{Methods}

Materials. G3P dehydrogenase (rabbit muscle), lactate dehydrogenase (rabbit muscle), triose phosphate isomerase (rabbit muscle), pyruvate kinase (rabbit muscle), horseradish peroxidase, G3P, ATP, glutathione, NAD(P)H and NAD(P) ${ }^{+}$were supplied by Boehringer Mannheim. D-GAD, DHAP, methylglyoxal, MTT and $o$-dianisidine were from Sigma. DHA was from Serva. D-Galacturonic acid was obtained from Fluka. Sephadex G-25 was obtained from Pharmacia LKB. Other reagents were from Merck and were of analytical grade.

Strains and culture conditions. Aspergillus nidulans strain WG096 (yA2 pabaAI) was used as wild-type with respect to carbon source utilization. The glycerol non-utilizing strains WG196 ( $y A 2$ pabaAl glcAl), WG197 (yA2 pabaAl glcB33), WG372 (yA2 pantoB100 glcC2) and glcD100 (pabaAl pantoA10 glcD100) were used. Strain WG216 (pabaAl cre $A^{d} l$ ) was used to study the effect of catabolite repression. Linkage group assignment was made using WG202 (biAl acrAl meaB6 pyroA4 pAl $s B 3$ nicB8 riboB2). The ethanol non-utilizing strains used were $\mathrm{Q} 8$ (alcR125), H542-500 (yA4 pyroA4 sB3 adE20 suadE20 alcA500 alcR500), $\mathrm{H} 444$ (biAl, alcR125), $\mathrm{H} 489$ (biAl nii4 aldA67), H352/7 (pabaAl alX4 alcA527) and WG379 (pabaAl alcA527 aldA67 ureAl).

For the preparation of conidiospores, $A$. nidulans was grown on complete medium (Pontecorvo et al., 1953) solidified with $1.2 \%(w / v)$ agar and containing $25 \mathrm{~mm}$-sucrose as carbon source. Mycelium was grown by inoculating $10^{6}$ spores $\mathrm{ml}^{-1}$ into minimal medium (Pontecorvo et al., 1953), supplemented with appropriate vitamins, with $50 \mathrm{~mm}$-D-fructose as carbon source, and incubating for $16 \mathrm{~h}$ at $37^{\circ} \mathrm{C}$, pH 6.0, in a New Brunswick Scientific orbital shaker ( 200 r.p.m.) using 1-litre flasks containing $300 \mathrm{ml}$. The mycelium was harvested by filtration and transferred to fresh minimal medium containing either glycerol (100 mM), DHA (100 mM), D-galacturonate (50 mM), Dglucose/glycerol $(25 \mathrm{~mm} / 100 \mathrm{mM})$, D-glucose $(50 \mathrm{mM})$ or D-fructose $(50 \mathrm{~mm})$. After $6 \mathrm{~h}$ incubation at $37^{\circ} \mathrm{C}$ in the orbital shaker, mycelium was again harvested by filtration, washed with cold saline and squeezed to remove excess fluid. The mycelium was then frozen with liquid nitrogen and stored at $-60^{\circ} \mathrm{C}$.

Growth tests were performed at $\mathrm{pH} 6.0$ as described previously (Uitzetter $e t$ al., 1986). The final concentrations of the carbon sources were: glycerol $100 \mathrm{mM}$, DHA $100 \mathrm{mM}$, D-galacturonate $50 \mathrm{mM}$, Dglucose/glycerol $25 \mathrm{~mm} / 100 \mathrm{mM}$, D-glucose $50 \mathrm{mM}$, D-fructose $50 \mathrm{mM}$. In other test plates using combinations of carbon sources, glycerol and DHA were used at a concentration of $100 \mathrm{mM}$, methylglyoxal at $10 \mathrm{mM}$ and $25 \mathrm{mM}, \mathrm{D}$-glucose, sucrose, D-fructose, D-galactose, lactose and Dxylose at $25 \mathrm{mM}$, acetate and L-glutamate at $100 \mathrm{mM}$.

Isolation and genetic mapping of three new glycerol mutants. A suspension of $10^{7}$ conidiospores from strain WG096 was irridiated for $30 \mathrm{~s}$ with a $30 \mathrm{~W}$ TUV lamp (Philips) at a dose of $2 \mu \mathrm{J} \mathrm{mm}^{-2} \mathrm{~s}^{-1}$. Mutants were enriched by filter enrichment, essentially as described by Uitzetter et al. (1986). DHA (100 mM) was used as carbon source. Three independent mutants were obtained. The three mutants were tested for complementation to each other and to the $g l c A 1$ and $g l c B 33$ mutations in heterokaryons and in diploids. The linkage groups of the three new mutants were determined by haploidization of diploids formed with strain WG202 (Upshall et-al., 1979). The three new mutants were designated $g l c E l, g l c F l$ and $g l c G 1$. The $g l c G l$ mutant was also tested for complementation to the glcD 100 mutation in heterokaryons and as diploid. The possible linkage of $g l c D 100$ and $g l c G l$, and of $g l c E l$ and glcFl, was investigated by meiotic recombination analysis. Standard genetical techniques were used (Pontecorvo et al., 1953; Clutterbuck, 1974).

Preparation of cell-free extracts. Cell-free extracts were prepared according to Arst et al. (1990).

Enzyme assays and protein determination. The activities of enzymes were measured with an Aminco DW-2 UV-VIS spectrophotometer using the double-beam mode at $340 \mathrm{~nm}$ versus $380 \mathrm{~nm}$ unless stated otherwise. Reactions were performed at $25^{\circ} \mathrm{C}$. D-GAD reductase $[\mathrm{NAD}(\mathrm{P}) \mathrm{H}]$ and $\mathrm{DHA}$ reductase $[\mathrm{NAD}(\mathrm{P}) \mathrm{H}]$ were assayed in $50 \mathrm{~mm}-$ sodium phosphate buffer pH 6.5, $0.2 \mathrm{mM}-\mathrm{NAD}(\mathrm{P}) \mathrm{H}, 5 \mathrm{~mm}-\mathrm{D}-\mathrm{GAD}$ or DHA. Glycerol dehydrogenase NAD(P) ${ }^{+}(\mathrm{EC} 1.1 .1 .6$ and 1.1.1.72) was assayed in $100 \mathrm{~mm}$-glycine/ $\mathrm{NaOH}$ pH 9.6, 0.4 mM-NAD(P)+, and $100 \mathrm{mM}$-glycerol. Alcohol dehydrogenase (ADH; EC 1.1.1.1) was assayed according to Creaser $e$ t al. (1985). DHA kinase activity was determined in $50 \mathrm{~mm}$-Tris/ $\mathrm{HCl} \mathrm{pH} 8.0,5 \mathrm{~mm}-\mathrm{MgCl}_{2}, 5 \mathrm{~mm}-\mathrm{ATP}$, $0.2 \mathrm{mM}-\mathrm{NADH}, 10 \mathrm{mM}-\mathrm{DHA}$ and 1 unit (U) G3P dehydrogenase. D-GAD kinase was assayed in $50 \mathrm{mM}-\mathrm{Tris} / \mathrm{HCl} \mathrm{pH} \mathrm{8.0,5} \mathrm{mm}-\mathrm{MgCl}_{2}$, 5 mM-ATP, 0.2 mM-NADH, $10 \mathrm{mM}$ D-GAD, 1 U G3P dehydrogenase and $1 \mathrm{U}$ triose phosphate isomerase. NAD-dependent $s n$-G3P dehydrogenase (EC 1.1.1.8) and FAD-dependent $s n$-G3P dehydrogenase (EC 1.1.99.5) were assayed as described previously (Arst $e t$ al., 1990). Glycerol oxidase was assayed according to Uwajima et al. (1980). Glycerol kinase (EC 2.7.1.30) was assayed in a discontinuous assay according to De Koning et al. (1987) and in a continuous assay in 50 mM-HEPES pH 7.0, 5 mM- $\mathrm{MgCl}_{2}, 10 \mathrm{~mm}$-glycerol, $0.1 \mathrm{~mm}$ NADH, 0.2 mm-phosphoenolpyruvate, $1 \mathrm{U}$ lactate dehydrogenase and $0.2 \mathrm{U}$ pyruvate kinase. Glyoxalase I (lactoylglutathione reductase: EC 4.4.1.5) was assayed according to Inoue et al. (1987). Glutathione reductase (EC 1.6.4.2) was assayed in $50 \mathrm{~mm}$-sodium phosphate buffer pH 6.5, 0.2 mM-NADPH and 2 mM-glutathione (ox.). Methylglyoxal dehydrogenase was assayed in $100 \mathrm{~mm}$-glycine/ $\mathrm{NaOH} \mathrm{pH} 9 \cdot 6$, $0.4 \mathrm{mM}-\mathrm{NAD}(\mathrm{P})^{+}$, and $2 \mathrm{mM}$-methylglyoxal.

Protein content in mycelial extracts was determined by the microbiuret method (Itzhaki \& Gill, 1964) using bovine serum albumin as a standard.

\section{Results}

Enzyme activities in wild-type A. nidulans after transfer to various carbon sources

To study the effect of different carbon sources on the level of enzymes involved in glycerol metabolism, wildtype $A$. nidulans and various mutants were grown on fructose and subsequently transferred to media with different carbon sources (see Methods). The transfer methodology enables mycelia at comparable growth stages to be compared. Ideally one would like to test, besides inducing and repressing carbon sources, also a non-repressing, non-inducing carbon source, in order to sort out induction and repression properly. In practice this cannot be realized since glycerol also arises from intermediary metabolism. Table 1 gives the data obtained with the wild-type strain WG096. In Fig. 1 a model for glycerol metabolism in A. nidulans is presented which is based on these activity measurements. 


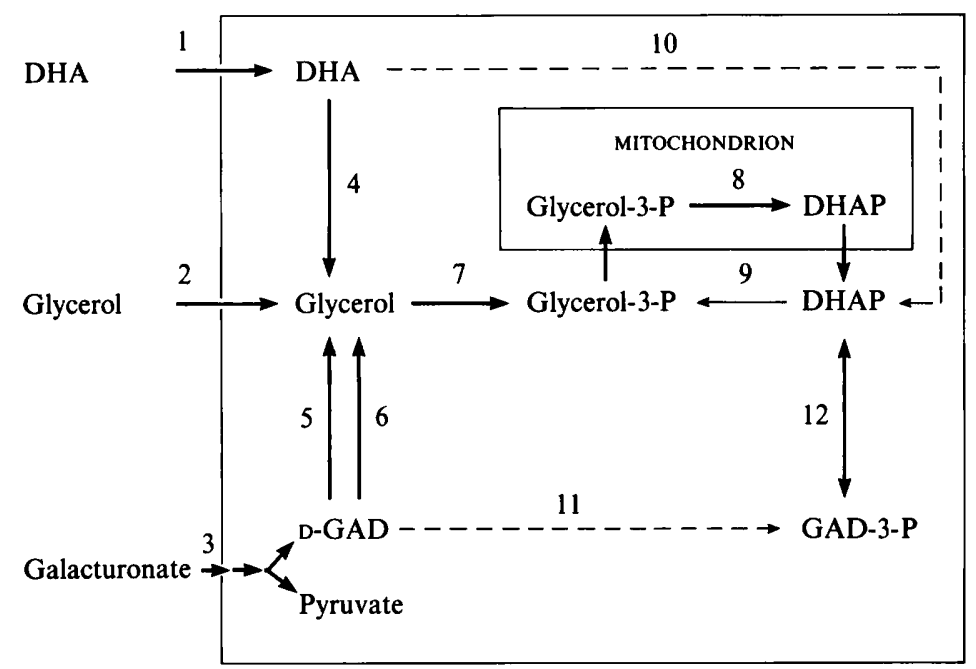

Fig. 1. Glycerol catabolism in $A$. nidulans. The following steps are indicated: 1, DHA uptake; 2, glycerol uptake; 3, D-galacturonate uptake and degradation to pyruvate and D-GAD; 4, NADP+. dependent glycerol dehydrogenase; 5, NADP+. dependent glycerol dehydrogenase; 6, ADH I; 7, glycerol kinase; 8, FAD-dependent G3P dehydrogenase; 9, NAD+-dependent G3P dehydrogenase; 10, DHA kinase; 11, D-GAD kinase; 12, triose phosphate isomerase.

Table 1. Enzyme activities in wild-type WG096 after transfer to medium containing various carbon sources

Mycelia were grown for $16 \mathrm{~h}$ in minimal medium with fructose and then transferred for $8 \mathrm{~h}$ to fresh minimal medium containing $100 \mathrm{~mm}$-glycerol (Glyc), $100 \mathrm{~mm}-\mathrm{DHA}, 50 \mathrm{mM}$ D-galacturonate (GalA), $25 \mathrm{~mm} / 100 \mathrm{mM}$-D-glucose/glycerol (Glc/Glyc), $50 \mathrm{mM}$-D-glucose (Glc) or $50 \mathrm{~mm}$-D-fructose (Fru). The specific activities shown are the means of two determinations, agreeing within $10 \%$ in each case. Data for glycerol kinase, G3P dehydrogenase (FAD), DHA reductase (NADPH) and D-GAD reductase (NADPH) were reported previously (Arst et al., 1990).

\begin{tabular}{|c|c|c|c|c|c|c|}
\hline \multirow[b]{2}{*}{ Enzyme } & \multicolumn{6}{|c|}{$\begin{array}{l}\left.\text { Activity [nmol } \min ^{-1}(\mathrm{mg} \text { protein })^{-1}\right] \\
\text { after transfer to: }\end{array}$} \\
\hline & Glyc & DHA & GalA & Glc/Glyc & Glc & Fru \\
\hline Glycerol kinase & 41 & 36 & 40 & 7 & - & - \\
\hline G3P dehydrogenase (FAD) & 53 & 45 & 32 & 8 & 9 & 10 \\
\hline G3P dehydrogenase (NAD) & 9 & 2 & 17 & 3 & 6 & 3 \\
\hline DHA reductase (NADPH) & 212 & 236 & 166 & 250 & 248 & 202 \\
\hline D-GAD reductase (NADPH) & 70 & 88 & 56 & 85 & 83 & 31 \\
\hline DHA reductase (NADH) & 17 & - & - & 3 & - & - \\
\hline D-GAD reductase (NADH) & 22 & - & 51 & 11 & - & 8 \\
\hline $\mathrm{ADH}$ & 107 & - & 278 & 8 & - & 20 \\
\hline Glycerol dehydrogenase (NADP) & 208 & 201 & 156 & 156 & 178 & 143 \\
\hline Glycerol dehydrogenase (NAD) & - & - & - & - & - & - \\
\hline DHA kinase & - & 19 & 15 & - & - & - \\
\hline D-GAD kinase & - & 3 & 2 & - & - & - \\
\hline Glycerol oxidase & - & - & - & - & - & - \\
\hline
\end{tabular}

- , Not detectable.

Upon transfer to glycerol, DHA or D-galacturonate, glycerol kinase (step 7 in Fig. 1) and mitochondrial G3P dehydrogenase (step 8) were induced. No glycerol kinase activity could be detected upon transfer to D-glucose or when refreshing the D-fructose medium. A low activity was found after transfer to a mixture of D-glucose and glycerol, indicating that glycerol kinase is subject to glucose catabolite repression. The level of mitochondrial G3P dehydrogenase increased about five-fold on glycerol, DHA or D-galacturonate as compared to cultures transferred to D-glucose, D-fructose or a mixture of Dglucose and glycerol. These data suggest that the flavoprotein is also subject to glucose catabolite repression. Under the culture conditions used here the catabolic pathway for glycerol is formed by glycerol kinase (step 7) and mitochondrial G3P dehydrogenase (step 8).

DHA reductase (NADPH) (step 4), D-GAD reductase (NADPH) (step 5) and glycerol dehydrogenase $\left(\mathrm{NADP}^{+}\right)$(reactions 4 and 5 in opposite direction) were 
present in fairly high amounts regardless of the carbon source used (Table 1). This is in good agreement with earlier studies in which a constitutive NADP+-dependent glycerol dehydrogenase from $\boldsymbol{A}$. nidulans was purified and characterized (Schuurink et al., 1990). The purified enzyme uses glycerol, DHA and D-GAD as substrates, the latter being less effective. As the activity ratios approximate those found with the purified enzyme it is very likely that the glycerol dehydrogenase $\left(\mathrm{NADP}^{+}\right)$activity, the DHA reductase (NADPH) activity and the D-GAD reductase (NADPH) activity found in crude extracts can be attributed to this single enzyme. Under physiological conditions the equilibrium will favour almost complete formation of glycerol.

Cytosolic G3P dehydrogenase $\left(\mathrm{NAD}^{+}\right)$(step 9) was present at low levels; D-galacturonate was the only carbon source upon which an elevated enzyme activity was found. DHA kinase (step 10) and D-GAD kinase (step 11) were both slightly induced on DHA and D-galacturonate. On other carbon sources no activity was observed. On glycerol and on D-galacturonate an NADH-specific D-GAD reductase (step 6) and an ADH activity were induced. No $\mathrm{NAD}^{+}$-dependent glycerol dehydrogenase activity or glycerol oxidase activity was detected. Various modifications ( $\mathrm{pH}$, temperature, addition of ions) to the standard assay procedures were tried without success.

\section{Involvement of alcohol dehydrogenase I in glycerol metabolism}

High ADH activities were found in wild-type mycelium after transfer to D-galacturonate or glycerol (Table 1). ADH I from $A$. nidulans uses a broad range of substrates, viz. a variety of aldehydes, alcohols and ketones (Creaser et al., 1985). Several of these compounds induce ADH I. We therefore tested the possibility that the NADHdependent $\mathrm{D}-\mathrm{GAD}$ reductase activity might be due to ADH I.
In $A$. nidulans a constant ratio between $\mathrm{ADH}$ and NADH-dependent D-GAD reductase activity of $5 \cdot 1 \pm 0 \cdot 6$ was found. Also a similar induction pattern for both enzyme activities was found in the wild-type and in the glycerol non-utilizing mutants. D-Galacturonate and glycerol were both inducers but glycerol was less effective (data not shown). When the wild-type strain was grown on ethanol $(300 \mathrm{mM})$ or on D-glucose/threonine $(4 \mathrm{~mm} / 50 \mathrm{~mm})$, high activities of both ADH [320 mU (mg protein $)^{-1}$ and $770 \mathrm{mU}$ (mg protein) ${ }^{-1}$ ] and NADHdependent $\mathrm{D}-\mathrm{GAD}$ reductase $\left[70 \mathrm{mU}(\mathrm{mg} \text { protein })^{-1}\right.$ and $165 \mathrm{mU}$ (mg protein) $\left.{ }^{-1}\right]$ were found. These media are well known to induce ADH I (Creaser et al., 1985).

In alc $A$ mutants, which have a defect in the structural ADH I gene (Pateman et al., 1983) and in alcR mutants, defective in a positive regulator gene (Sealy-Lewis \& Lockington, 1984), no NADH-dependent D-GAD reductase activity could be detected after transfer to Dgalacturonate or glycerol, whereas in aldA (Pateman et $a l ., 1983)$ mutants, deficient in the aldehyde dehydrogenase structural gene, normal induction of $\mathrm{ADH}$ and NADH-dependent D-GAD reductase was found on these two media. Therefore we conclude that the NADH-dependent D-GAD reductase activities found are attributable to $\mathrm{ADH} \mathrm{I}$.

alc $A$, alcR, ald $A$, alc $A$ alc $R$ and alc $A$ ald $A$ mutants showed normal growth on solid media with $\mathrm{D}$-galacturonate or glycerol as carbon source. ADH I, and therefore NADH-dependent D-GAD reductase, is apparently not necessary for fungal growth on D-galacturonate or glycerol, almost certainly due to the high levels of the constitutive NADPH-dependent D-GAD reductase.

\section{Growth characteristics of glycerol non-utilizing mutants}

The growth characteristics of seven different glycerol mutants of $A$. nidulans are presented in Table 2. The glcA mutant was postulated to be defective in glycerol kinase on the basis of growth characteristics (Payton, 1978).

Table 2. Growth characteristics of A. nidulans glycerol mutants

Glycerol and DHA were present at $100 \mathrm{~mm}$, D-galacturonate and D-glucose at $50 \mathrm{mM}$. In combinations $25 \mathrm{mM}-\mathrm{D}$-glucose was used with $100 \mathrm{mM}$-glycerol or DHA, or $50 \mathrm{mm-D}$-galacturonate. Tests were performed at $37^{\circ} \mathrm{C} .++++$, Good growth; +++ , moderate; + , very poor; -, no growth.

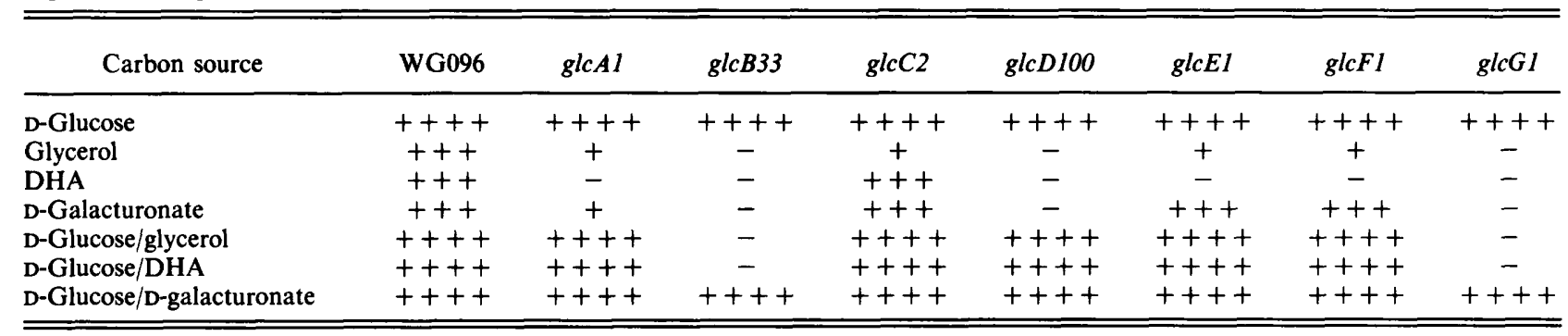


Table 3. Glycerol kinase and mitochondrial G3P dehydrogenase (FAD) activities in wild-type and mutant strains of $A$. nidulans

\begin{tabular}{|c|c|c|c|c|c|c|}
\hline \multirow[b]{2}{*}{ Strain } & \multicolumn{6}{|c|}{ Activity $\left[\mathrm{nmol} \min ^{-1}(\mathrm{mg} \text { protein })^{-1}\right]$ after transfer to: } \\
\hline & Glycerol & DHA & Galacturonate & $\begin{array}{l}\text { Glucose/ } \\
\text { glycerol }\end{array}$ & Glucose & Fructose \\
\hline \multicolumn{7}{|c|}{ (a) Glycerol kinase } \\
\hline WG096 & 41 & 36 & 40 & 7 & - & - \\
\hline$g l c A$ & - & - & - & - & - & - \\
\hline$g l_{c} B$ & 33 & 40 & 31 & 2 & - & - \\
\hline glcC & 10 & 80 & 48 & - & - & - \\
\hline$g l c D$ & 51 & 70 & 50 & - & - & - \\
\hline$g l c E$ & 35 & 43 & 38 & - & - & - \\
\hline$g l c F$ & 29 & 32 & 47 & 3 & - & - \\
\hline$g l c G$ & 64 & 31 & 50 & 5 & - & - \\
\hline creA & 85 & 48 & 52 & 80 & 8 & 10 \\
\hline alc $A$ & 38 & 45 & 27 & 2 & - & - \\
\hline \multicolumn{7}{|c|}{ (b) G3P dehydrogenase $(F A D)$} \\
\hline WG096 & 53 & 45 & 32 & 8 & 9 & 10 \\
\hline glcA & 70 & 90 & 139 & 31 & 9 & 7 \\
\hline$g l c B$ & - & - & - & - & - & - \\
\hline glcC & 5 & 81 & 21 & 11 & 9 & 6 \\
\hline$g l c D$ & - & 13 & 2 & - & - & - \\
\hline$g l c E$ & 15 & 59 & 19 & 11 & 5 & 4 \\
\hline$g l c F$ & 11 & 15 & 42 & 12 & 8 & 6 \\
\hline$g l c G$ & 5 & 7 & 10 & 11 & 4 & 4 \\
\hline creA & 18 & 47 & 23 & 30 & 11 & 7 \\
\hline alc $A$ & 10 & 19 & 2 & 3 & 3 & 2 \\
\hline
\end{tabular}

-, Not detectable.

Table 3 presents biochemical evidence which confirms that this mutant lacks glycerol kinase activity. In the glcB33 mutant no mitochondrial G3P dehydrogenase activity was found. The $g l c C 2$ mutant was impaired in glycerol uptake (Visser et al., 1988). The growth features of the glcD100 mutant (Arst et al., 1990) and three new glycerol mutants, $g l c E 1, g l c F 1$ and $g l c G 1$, are also presented. The growth characteristics of the glcGI mutant were similar to those of the glcB33 mutant. The G3P dehydrogenase (FAD) activity measured in this mutant was always very low (Table 3). The glcB33 mutation, located on chromosome I, and the glcGl mutation, located on chromosome VIII, complemented each other both in a heterokaryon and in a diploid. Since all other enzyme levels in this mutant are comparable to those of the wild-type on each of the carbon sources tested (data not shown), it is probable that the glcGl mutant, like the glcB33 mutant, is defective in the mitochondrial G3P dehydrogenase activity. In the glcD100 mutant FAD-dependent G3P dehydrogenase activities are also greatly decreased (Arst et al., 1990). The glcD100 mutation was found to be located on chromosome VIII (Arst et al., 1990). Complementation both in a heterokaryon and in a diploid together with free recombination in meiosis demonstrated that $\mathrm{glcD} 100$ and $g l c G l$ are non-allelic.

The $g l c E l$ and $g l c F l$ mutations have been allocated to chromosome VI. They complement each other, as well as the $g l c A 1, g l c B 33$ and $g l c C 2$ mutations, in both heterokaryons and diploids.

Effects of carbon source on glycerol kinase and mitochondrial G3P dehydrogenase activities in glycerol mutants

When the glycerol uptake mutant $(\mathrm{glcC} 2)$ was transferred to glycerol no appreciable induction of glycerol kinase and G3P dehydrogenase occurred. Upon transfer to DHA or D-galacturonate, both enzymes were induced to levels comparable to those found in the wild-type WG096. Glycerol uptake or glycerol formation is therefore a likely prerequisite for induction of glycerol kinase and G3P dehydrogenase.

G3P dehydrogenase activity was elevated in the glycerol kinase mutant ( $g l c A 1$ ) upon transfer of my- 
celium to glycerol, DHA, D-galacturonate or D-glucose/ glycerol. However, G3P levels remained low in this mutant under these conditions (D. H. A. Hondmann \& J. Visser, unpublished results), indicating that in $A$. nidulans G3P is unlikely to be the direct inducer of glycerol catabolism. In Escherichia coli G3P has been reported to be the inducer for transcription of the genes involved in glycerol metabolism (Cozarelli et al., 1968).

In both the $g l c B 33$ and $g l c G 1$ mutants glycerol kinase activities were comparable to wild-type levels. G3P accumulated in the glcB33 mutant when D-fructosegrown mycelium was transferred to glycerol, DHA, Dgalacturonate or even to D-glucose/glycerol (J. Visser and others unpublished results). G3P was therefore unlikely to result in feedback inhibition, or to repress transcription, of glycerol kinase.

The $g l c E 1$ and $g l c F 1$ mutations had no pronounced effects on glycerol kinase or G3P dehydrogenase activities. In the $g l c E 1$ mutant G3P dehydrogenase was not induced to the same extent as in the wild-type upon transfer to glycerol or D-galacturonate. G3P dehydrogenase activity remained low in the $g l c F 1$ mutant transferred from a glycolytic carbon source to glycerol or DHA.

The ADH I defective strain (alcA527) failed to induce G3P dehydrogenase when it was transferred to Dgalacturonate. This can be interpreted as an additional line of evidence that ADH I is involved in D-GAD catabolism. On test plates alcA mutants grew as well as the wild-type on D-galacturonate. In this case a transfer of $6 \mathrm{~h}$ to a liquid medium was not comparable to growth on a solid medium for $2-3 \mathrm{~d}$.

Catabolite repression affected both glycerol kinase and G3P dehydrogenase, as could be concluded from the enzyme levels found in a cre $A^{d}-1$ mutant. Neither enzyme was repressed in this mutant on a mixed carbon source consisting of D-glucose and glycerol. Carbon catabolite repression cannot be the only regulatory mechanism, since no induction was found in the $c r e A^{d}-1$ mutant when transferred to D-glucose or D-fructose. It is apparent that glycerol, or a derivative, must act as an inducer.

The remaining enzyme levels measured in this study indicated no gross differences between the wild-type and the various mutants, with the exception that DHA kinase and D-GAD kinase activities were found to be induced to levels about fivefold higher on DHA and on D-galacturonate in the case of the glycerol kinase mutant ( $g l c A l)$ as compared to the wild-type.

\section{Further characterization of glcE1 and glcFI}

Since the $g l c E 1$ and $g l c F 1$ mutants demonstrated no obvious enzyme lesions, we investigated the possibility that the poor growth of these two mutants on DHA and glycerol could be a result of a toxic effect. In Saccharo- myces cerevisiae and $E$. coli mutants have been described that produce methylglyoxal during glycerol catabolism and are therefore subject to glycerol toxicity (Cooper \& Anderson, 1970; Penninckx et al., 1983). The nonenzymic formation of methylglyoxal from glyceraldehyde under physiological conditions has also been reported (Riddle \& Lorenz, 1968). Several enzymes that were reported to be involved in methylglyoxal metabolism of various micro-organisms (Cooper, 1984; Thornally, 1990) were assayed in wild-type $A$. nidulans and in mycelium of the $g l c E l$ and $g l c F 1$ mutants which was transferred to DHA or to glycerol. All enzymes tested showed comparable activities in all three strains or were undetectable. Glyoxalase I activities of $15-30 \mathrm{mU}(\mathrm{mg}$ protein $)^{-1}$ were measured; NADPH-dependent methylglyoxal dehydrogenase activities were in the range 30 $50 \mathrm{mU}$ (mg protein $)^{-1}$ in all strains. No NADHdependent methylglyoxal dehydrogenase was detected. Glutathione reductase activities were in the range 20$40 \mathrm{mU}(\mathrm{mg} \text { protein })^{-1}$.

On solid media containing D-glucose, sucrose, Dfructose, acetate, D-galactose lactose, L-glutamate or Dxylose, to which DHA, glycerol or methylglyoxal were added, no growth differences between the wild-type and glcEl or $g l c F 1$ were observed, suggesting that the growth defects of $g l c E l$ and $g l c F l$ mutants are not due to toxicity.

\section{Discussion}

In Aspergillus nidulans both biochemical and genetical evidence indicates that the main route for glycerol, DHA and D-GAD catabolism involves glycerol kinase and mitochondrial G3P dehydrogenase (FAD), and therefore depends on respiration. At least two regulatory mechanisms are important for glycerol catabolism. Firstly there is a pathway-specific induction as glycerol kinase and the FAD-dependent G3P dehydrogenase are induced by glycerol (and by DHA and D-galacturonate), whereas other enzymes that could have formed an alternative route were apparently uninduced. Secondly glucose catabolite repression affects the induction of glycerol kinase and FAD-dependent G3P dehydrogenase. This repression was not absolute as low levels of both enzymes are found using mixed substrates, e.g. glucose/ glycerol. In the cre $A^{d}-1$ mutant transferred to D-glucose or D-fructose, uninduced levels of glycerol kinase and G3P dehydrogenase activities are found, whereas pathway-specific induction is prominent on a mixture of $D$ glucose and glycerol. The two mechanisms make it possible for $\boldsymbol{A}$. nidulans to control the amount of glycerol present intracellularly in a flexible way. If glucose repression were too tight this would interfere with the 
need to catabolize glycerol in the presence of repressing carbon sources, e.g. under conditions where the osmotic value of the environment drops.

Upon uptake DHA will be largely converted to glycerol by the NADPH-dependent DHA reductase. The properties of the purified enzyme showed that glycerol formation is favoured under physiological conditions (Schuurink et al., 1990). In vivo natural abundance ${ }^{13} \mathrm{C}$ NMR studies show that $A$. nidulans wildtype and glycerol non-utilizing mutants grown on, or transferred to, DHA accumulate glycerol under aerobic conditions. DHA kinase was detected at low levels in the wild-type but was elevated fivefold in the glycerol kinase mutant. An $A$. niger glycerol kinase mutant could grow on DHA (Witteveen et al., 1990), whereas the equivalent $A$. nidulans mutant could not. This is a major difference in glycerol catabolism between these two fungi. In $A$. niger DHA kinase formed an effective bypass which apparently did not function, under the conditions used here, in $A$. nidulans. We believe that the balance between reduction and phosphorylation of DHA is very much in the direction of reduction in $A$. nidulans. DHA kinase and D-GAD activities could be attributed to a single enzyme in $A$. niger (Witteveen et al., 1990). In A. nidulans both activities are also likely to be due to a single enzyme. Both activities appear simultaneously on DHA and on Dgalacturonate and in the same ratio. DHA kinase may nevertheless be of physiological importance in $A$. nidulans for growth on DHA or D-galacturonate, when the anabolic reduction charge is low or when oxygen levels are low. This is supported by the observation that the glcAI mutant does grow under microaerophilic conditions.

D-Galacturonate was reported to be degraded to pyruvate and a triose, probably D-GAD (Uitzetter, 1982). The induction of D-GAD reductase (NADH) upon transfer to D-galacturonate confirmed this prediction. D-GAD formed from D-galacturonate will be almost completely converted to glycerol by ADH I or by NADPH-dependent D-GAD reductase activity catalysed by the well-characterized DHA reductase. In Schizosaccharomyces pombe NADH-dependent D-GAD reductase and ADH I were also activities of the same enzyme (Kong et al., 1985). The induction in A. nidulans of ADH I upon growth on D-galacturonate indicates an unexpected physiological role for this enzyme. When grown on cell walls of higher plants, $A$. nidulans excretes pectic enzymes, amongst others. One of the major endproducts formed from pectin is D-galacturonate (Pilnik \& Rombouts, 1981). Since both D-GAD reductases are found at comparable levels on D-galacturonate, and because both enzymes have comparable $K_{\mathrm{m}}$ values for D-GAD (Creaser et al., 1985; Schuurink et al., 1990), the balance of fluxes through the two enzymes will be mainly controlled by the anabolic and catabolic reduction charge. The second major difference with $A$. nidulans is that in $A$. niger an NADPH-dependent DGAD reductase is induced on D-galacturonate instead of ADH I (Witteveen et al., 1990).

Schuurink et al. (1990) proposed the existence of a 'glycerol cycle', composed of DHAP phosphatase, NADPH-dependent DHA reductase, glycerol kinase and FAD-dependent G3P dehydrogenase. An operating glycerol cycle would regenerate $\mathrm{NADP}^{+}$and generate ATP under conditions when excess NADPH is formed. Under the growth conditions used here the glycerol cycle does not seem to be used, since no glycerol kinase activity can be found in mycelium transferred to D-glucose or Dfructose.

The cytosolic NAD ${ }^{+}$-dependent G3P dehydrogenase is present at basal levels in all mycelia. This enzyme seems to be able to maintain appropriate G3P levels necessary for lipid biosynthesis. This may explain why glycerol non-utilizing mutants do not show an aberrant colonial morphology.

Three independent glycerol non-utilizing mutants, $g l c B 33, g l c D 100$ and $g l c G 1$, were found to be affected in the mitochondrial G3P dehydrogenase activity. In E. coli the FAD-dependent G3P dehydrogenase is composed of three different subunits, a catalytic subunit, a subunit that shuttles reduction equivalents to the electron chain, and an anchor subunit (Cole et al., 1988). If in A. nidulans this enzyme has a similar composition, as suggested by the three complementation groups found, the glcB33 mutation is most likely to affect the catalytic subunit, since no activity can be detected in this mutant. The glcD 100 mutant can grow on mixed carbon sources like D-glucose/glycerol, whereas the glcG1 mutant cannot. Therefore, inhibition of growth due to G3P accumulation does not seem to occur in the glcD100 mutant. This can be explained by assuming that the $g l c D 100$ mutation affects the anchor subunit, leading to a partially active enzyme that is not properly bound to the inner mitochondrial membrane, and therefore only found in low activities in the mitochondrial cell-free extract. The glcGl mutation will, in this model, disrupt the subunit that shuttles reduction equivalents from the catalytic subunit to the respiratory chain.

On D-glucose/D-galacturonate test plates glcB33 and glcGl strains grow like the wild-type, whereas they do not grow on D-glucose/glycerol or D-glucose/DHA. Glucose catabolite repression is much more restrictive for one or more steps in the uptake and degradation of $D-$ galacturonate to D-GAD and pyruvate than it is for the uptake of DHA and glycerol and for glycerol kinase induction.

The true nature of the $g l c E 1$ and $g l c F 1$ mutations remains unresolved. Involvement of a DHA uptake 
system seems unlikely since all enzymes involved in glycerol metabolism were found at normal or slightly diminished levels after transfer to DHA or glycerol. It is also unlikely that these mutants have an impaired detoxification system.

We thank Dr Clive Roberts (University of Leicester) for supplying the original $g l c A l$ and $g l c B 33$ mutants and Dr H. M. Sealy-Lewis (University of Hull) for ethanol non-utilizing strains. Peter Kolster is thanked for his contributions in this study. We thank Professor Herb Arst for his comments on the manuscript. This investigation was supported by the Foundation for Fundamental Biological Research (BION) which is subsidized by the Netherlands Organization for Scientific Research (NWO) (grant no. 811-419-261).

\section{References}

ARST, H. N., HondmanN, D. H. A. \& Visser, J. (1990). A translocation activating the cryptic nitrogen regulation gene are $B$ inactivates a previously unidentified gene involved in glycerol utilisation in Aspergillus nidulans. Molecular and General Genetics 223, 134-137.

BeEver, R. E. \& LARACY, E. P. (1986). Osmotic adjustment in the filamentous fungus Aspergillus nidulans. Journal of Bacteriology 168 $1358-1365$

ClutterbucK, A. J. (1974). Aspergillus nidulans. In Handbook of Genetics, vol. 1, pp. 447-510. Edited by R. C. King. New York: Plenum Press.

Cole, S. T., Eiglmeier, K., Ahmed, S., Honore, N., Elmes, L., ANDERSON, W. F. \& WEINER, J. H. (1988). Nucleotide sequence and gene-polypeptide relationships of the $g l p A B C$ operon encoding the anaerobic sn-glycerol-3-phosphate dehydrogenase of Escherichia coli K-12. Journal of Bacteriology 170, 2448-2456.

COOPER, R. A. (1984). Metabolism of methylglyoxal in microorganisms. Annual Review of Microbiology 38, 49-68.

COOPER, R. A. \& ANDERSON, A. (1970). The formation and catabolism of methylglyoxal during glycolysis in Escherichia coli. FEBS Letters 11, 273-276.

Cozarelli, N. R., Koch, J. P., Hayashi, S. \& Lin, E. C. C. (1968). Growth stasis by accumulated L- $\alpha$-glycerophosphate in Escherichia coli. Journal of Bacteriology 90, 1325-1329.

Creaser, E. H., Porter, R. L., Britt, K. A., Pateman, J. A. \& Doy, C. H. (1985). Purification and preliminary characterization of alcohol dehydrogenase from Aspergillus nidulans. Biochemical Journal 225, 449-454.

De Koning, W., Harder, W. \& Dijkhuizen, L. (1987). Glycerol metabolism in the methylotrophic yeast Hansenula polymorpha: phosphorylation as the initial step. Archives of Microbiology 148, 314320.

DiJkema, C., Kester, H. C. M. \& Visser, J. (1985). ${ }^{13}$ C NMR studies of carbon metabolism in the hyphal fungus Aspergillus nidulans. Proceedings of the National Academy of Sciences of the United States of America 82, 14-18.

inoue, Y, Rhee, H.-I., Watanabe, K., Murata, K. \& Kimura, A. (1987). Metabolism of 2-ketoaldehydes in mold: purification and characterization of gyoxylase I from Aspergillus niger. Journal of Biochemistry 102, 583-589.

ITZHAKI, R. F. \& Gill, O. M. (1964). A microbiuret method for estimating proteins. Analytical Biochemistry 9, 401-410.

Kong, Y.-C., MaY, J. W. \& Marshall, J. H. (1985). Glycerol oxidation and triose reduction by pyridine nucleotide-linked enzymes in the fission yeast Schizosaccharomyces pombe. Journal of General Microbiology 131; 1571-1579.
LEgISA, M. \& MATTEY, M. (1986). Glycerol as an initiator of citric acid accumulation in Aspergillus niger. Enzyme and Microbial Technology 8, 258-259.

LIN, E. C. C. (1976). Glycerol dissimilation and its regulation in bacteria. Annual Review of Microbiology 30, 535-578.

LiN, E. C. C. (1977). Glycerol utilization and its regulation in mammals. Annual Review of Biochemistry 46, 765-795.

MaY, J. W. \& Sloan, J. (1981). Glycerol utilization by Schizosaccharomyces pombe: dehydrogenation as the initial step. Journal of General Microbiology 123, 183-185.

Pateman, J. A., Doy, C. H., Olson, J. E., Norris, U., Creaser, E. H. \& HYNES, M. (1983). Regulation of alcohol dehydrogenase (ADH) and aldehyde dehydrogenase (AldDH) in Aspergillus nidulans. Proceedings of the Royal Society B217, 243-264.

Payton, M. A. (1978). A genetic study of sugar metabolism and transport in Aspergillus nidulans. PhD thesis, University of Leicester, UK.

Penninckx, M. J., Jaspers, C. J. \& Legrain, M. J. (1983). The glutathione-dependent glyoxylase pathway in the yeast Saccharomyces cerevisiae. Journal of Biological Chemistry 258, 6030-6036.

PilNIK, W. \& Rombouts, F. M. (1981). Pectic enzymes. In Enzymes and Food Processing, pp. 105-128. Edited by G. G. Birch, N. Blackbrough and K. J. Parker. London: Applied Science Publishers.

PonteCorvo, G., Roper, J. A., Hemmons, L. M., MCDonald, K. D. \& Bufton, A. W. J. (1953). The genetics of Aspergillus nidulans. Advances in Genetics 5, 141-239.

RIDDLE, V. \& LORENZ, F. W. (1968). Nonenzymic, polyvalent anioncatalyzed formation of methylglyoxal as an explanation of its presence in physiological systems. Journal of Biological Chemistry 243, 2718-2724.

SchuUrink, R., Busink, R., HondmanN, D. H. A., Witteveen, C. F. B. \& VISSER, J. (1990). Purification and properties of NADP+ dependent glycerol dehydrogenases from Aspergillus nidulans and $A$. niger. Journal of General Microbiology 136, 1043-1050.

Sealy-Lewis, H. M. \& Lockington, R. A. (1984). Regulation of two alcohol dehydrogenases in Aspergillus nidulans. Current Genetics 8, 253-259.

ThORNALly, P. J. (1990). The glyoxalase system: new developments towards functional characterization of a metabolic pathway fundamental to biological life. Biochemical Journal 269, 1-11.

UITZETTER, J. H. A. A. (1982). Studies on carbon metabolism in wild type and mutants of Aspergillus nidulans. PhD thesis, Agricultural University Wageningen, The Netherlands.

UITZETTER, J. H. A. A., Bos, C. J. \& VisSER, J. (1986). Characterization of Aspergillus nidulans mutants in carbon metabolism isolated after Dgalacturonate enrichment. Journal of General Microbiology 132, $1167-1172$.

Upshall, A., Giddings, B., Teow, S. C. \& Mortimore, I. D. (1979). Novel methods for genetic analysis in fungi. In Proceedings of the Third International Congress on the Genetics of Industrial Microorganisms, pp. 197-204. Edited by O. K. Sebek \& A. Laskin.

Uwajima, T., Akita, H., Ito, K., Mihara, A., Aisaka, K. \& Terada, O. (1980). Formation and purification of a new enzyme, glycerol oxidase and stoichiometry of the enzyme reaction. Agricultural and Biological Chemistry 4, 399-406.

VAN LAERE, A. J. \& Hulsmans, E. (1987). Water potential, glycerol synthesis, and water content of germinating Phycomyces spores. Archives of Microbiology 147, 257-262.

VisSer, J., Van Rooijen, R., DiJkema, C., Swart, K. \& Sealy-Lewis, H. M. (1988). Glycerol uptake mutants of the hyphal fungus Aspergillus nidulans. Journal of General Microbiology 134, 655-659.

Viswanath-Reddy, M., Pyle, J. E. \& Howe, H. B. (1978). Purification and properties of NADP+-linked glycerol dehydrogenase-from Neurespora crassa. Journal of General Microbiology 107, 289296.

Witteveen, C. F. B., Van de Vondervoort, P., Dijkema, C., Swart, K. \& VisSER, J. (1990). Characterization of a glycerol kinase mutant in Aspergillus niger. Journal of General Microbiology 136, 1299-1305. 